Article

\title{
An Implementation Scheme of Range and Angular Measurements for FMCW MIMO Radar via Sparse Spectrum Fitting
}

\author{
Lidong Huang ${ }^{1}$, Xianpeng Wang ${ }^{1, *}$, Mengxing Huang ${ }^{1, *}$, Liangtian Wan ${ }^{2}$, Zhiguang Han ${ }^{1}$ and \\ Yongqin Yang ${ }^{1}$ \\ 1 State Key Laboratory of Marine Resource Utilization in South China Sea and School of Information and \\ Communication Engineering, Hainan University, Haikou 570228, China; \\ huanglidong@hainanu.edu.cn (L.H.); hanzhiguang@126.com (Z.H.); yangyq@hainu.edu.cn (Y.Y) \\ 2 Key Laboratory for Ubiquitous Network and Service Software of Liaoning Province, School of Software, \\ Dalian University of Technology, Dalian 116620, China; wanliangtian@dlut.edu.cn \\ * Correspondence: wxpeng2016@hainanu.edu.cn (X.W.); huangmx09@hainanu.edu.cn (M.H.)
}

Received: 2 February 2020; Accepted: 22 February 2020; Published: 27 February 2020

\begin{abstract}
The work presented in this paper is about implementing a frequency-modulated continuous wave (FMCW) multiple-input multiple-output (MIMO) positioning radar and a sparse spectrum fitting (SpSF) algorithm for range and angular measurements. First, we designed a coherent FMCW MIMO radar system working in the S-band with low power consumption that consists of four transmitter and four receiver antennas and has the ability to extend its virtual aperture; thus, this system can achieve a higher resolution than conventional phased array radars. Then, the SpSF algorithm was designed for estimating the distance and angle of the targets in the FMCW MIMO radar. Due to the fact that the SpSF algorithm can exploit the spatial sparsity diversity of a signal, the SpSF algorithm that is applied in the designed MIMO radar system can achieve a better estimation performance than the multiple signal classification (MUSIC) and Capon algorithms, especially in the context of small snapshots and low signal-to-noise ratios (SNRs). The simulated and experimental results are used to prove the effectiveness of the designed MIMO radar and the superior performance of the algorithm.
\end{abstract}

Keywords: FMCW; MIMO radar; sparse spectrum fitting; position measurement

\section{Introduction}

Since the concept of a multiple-input multiple-output (MIMO) radar [1] was proposed in 2004, it has attracted large amounts of attention of researchers. As in MIMO communication systems, multiple transmitting antennas and multiple receiving antennas are arranged in an MIMO array system to improve the accuracy of angular measurement [2,3]. Benefitting from the equivalent virtual array principle [4], an MIMO radar has the ability to identify more targets and to get a higher azimuth resolution than traditional phased array radars. Because that the frequency-modulated continuous wave (FMCW) signals have the ranging ability and low power consumption [5], they are widely used in MIMO radar systems. As a method that is relatively easier to implement than frequency division multiplexing structure [6], the time division multiplexing (TDM) structure [7] is widely used in MIMO radar systems. This structure realizes the orthogonality of the waveform by switching the transmitting and receiving channels at different times.

With a large number of theoretical results [8-10] in MIMO radars, some MIMO radar systems [11-15] have been implemented for the experimental verification of related technologies. In reference [11], a through-wall MIMO imaging radar system that generated the real-time imaging of a target at a 
frame rate of $0.5 \mathrm{~Hz}$ and that operated at a stand-off range of $6 \mathrm{~m}$ or more was proposed. The 44 virtual antennas with half-wavelength element-to-element spacing in uniform linear array (ULA) were formed by using a time division multiplexing technique with eight real receive elements and 13 transmit elements that could produce 2D (range vs. cross-range) image. A demonstration of an MIMO over-the-horizon radar was presented in [12]. This system created the beamforming of the transmitter signal on a multi-element array and used a trans-horizon radar receiver to receive the target echo. After using space-time signal processing, the results showed that the ability of the MIMO radar to suppress spatial clutter was improved by $35 \mathrm{~dB}$, a result that verified the superior performance of the MIMO system. These radars are relatively large and have high power consumption. On the other hand, some small MIMO radars have been designed [6,13,14]. In [6], a prototype automotive radar system was able to simultaneously generate multiple transmit beams. The system was based on a four-channel $77 \mathrm{GHz}$ FMCW radar sensor, and the measured beam pattern of multiple, simultaneously generated beams were in good accordance with simulations. Similarly, a 77 GHz FMCW MIMO radar was implemented for 2D target localization by using an SiGe single-chip transceiver in [13]. Radar measurements were performed by using the digital-beamforming method with a $10 \mathrm{GHz}$ modulation bandwidth to show the applicability of a highly miniaturized two-channel radar in [14]. However, the monolithic microwave-integrated circuits (MMIC) MIMO radar is limited in size and can only obtain fewer virtual array elements.

In terms of MIMO radar positioning algorithms, most practical radars use conventional beamforming $(\mathrm{CBF})$ to estimate the distance and angle of targets. This approach is easy to implement, but its resolution is limited by the Rayleigh criterion [15], and the bandwidth [16] of the radar system is not satisfactory. In reference [17], a 2D multiple signal classification (MUSIC) algorithm for the joint estimation of the angle and distance was presented. The algorithm was analyzed with respect to its application to coherent MIMO arrays, and it was able to achieve a good performance on the experimental data scenarios. However, there are many estimation algorithms [18-24] with excellent performances that are only based on the simulation data, and there is no experimental system to verify them. For example, a signal model of a monostatic MIMO sparse frequency FMCW radar was established in [21], and a modified 2D iterative adaptive approach (IAA) algorithm for range-azimuth processing was presented and verified with simulated data. A sparse spectrum fitting (SpSF) algorithm for the estimation of direction of arrivals (DOAs) of multiple sources was introduced in [23], and the method of signal power estimation was proposed in [24]. Simulation examples were presented to illustrate several performance characteristics of the technique in the context of low signal-to-noise ratios (SNRs) and small snapshots.

In order to achieve a feasible MIMO radar system for performing the range and angular estimation algorithm, the contribution of this paper can be summarized as follows:

1. A small engineering FMCW MIMO radar system is implemented with four transmitter and four receiver antennas by using the time division multiplexing (TDM) technique.

2. Based on experiment and real data, a sparse spectrum fitting (SpSF) algorithm is designed for estimating the distance and angle of a target via the use of the the sparsity of signals, which verify not only the effectiveness of the designed MIMO radar system but also the superior performance of the algorithm.

The paper is organized as follows: Section 2 describes the signal model of the FMCW MIMO radar, the implemented FMCW MIMO radar system is described in Section 3, the SpSF algorithm is described in Section 4, the simulation and experimental results are presented in Section 5; and Section 6 draws the conclusions. 


\section{FMCW MIMO Signal Model}

When the FMCW signal is used for the transmission signal of a radar system, its expression for a sweep period can be written as:

$$
s(t)=V_{T} \cos \left(2 \pi f_{c} t+\pi k t^{2}+\varphi_{0}\right), 0 \leq t \leq T,
$$

where $V_{T}$ and $f_{\mathcal{C}}$ represent the amplitude and the start frequency of the chirp sweeping, respectively; $k=B / T$, where $B$ denotes the transmitted bandwidth and $T$ is the sweep duration; and $\varphi_{0}$ is the initial phase of the signal.

In the case of ignoring the addition of noise while assuming that a far-field target scatters the signal, the echo signal received by the receiving elements is expressed as:

$$
r(t)=A s(t-\tau),
$$

where $A$ is the sum of the receiving element gains and the propagation loss of the signal, and $\tau$ is the time delay of the signal scattering from the transmitting element to the target and then back to the receiving element, which contains the range and angle information of the target.

Under the assumption of a narrowband far-field signal source, there is an approximate relationship such that:

$$
s(t-\tau) \approx s(t) e^{-j w_{0} \tau},
$$

Then, the received echo is mixed with the transmitting signal like $r(t) \times s(t)$, and it can be filtered out of the high frequency component; then, the intermediate frequency (IF) signal can be obtained and expressed as:

$$
u(t)=V \cos \left[2 \pi k \tau t+2 \pi f_{c} \tau\left(1-\frac{\tau k}{2 f_{c}}\right)\right],
$$

where $V$ is the amplitude of the IF signal. According to the definition of $k$, it has the following relationship $\left(\tau k / 2 f_{c}\right) \ll 1$. Then, there is an approximate relationship, which is shown as:

$$
u(t) \approx V \cos \left[2 \pi k \tau t+2 \pi f_{c} \tau\right],
$$

Then, the IF signal is discretely sampled, and the sampling frequency is assumed to be $f_{s}$. The sampled discrete signal can be expressed as:

$$
u(n)=V \cos \left(2 \pi k \tau n T_{s}+2 \pi f_{c} \tau\right), n=0,1,2, \cdots, L-1,
$$

where $T_{S}=1 / f_{S}$ is the sampling time and $L=T f_{s}$ is the total number of sampling points.

In an MIMO radar system model, it is assumed that there are $K$ far-field targets and $M$ equivalent receiving array elements, and these array elements are generally arranged into a uniform linear array with equal intervals. Assuming that the gain of each array element is the same, the echo signal received by the $m$-th array element (after mixing and sampling) can be expressed as:

$$
x_{m}(n)=\sum_{k=1}^{K} V_{k} \cos \left(2 \pi \frac{B}{T} \tau_{k} n T_{s}+2 \pi f_{c} \tau_{k}\right)+w_{m}(n), n=0,1,2, \cdots, L-1,
$$

where $w(n)$ is the discrete noise and $x(n)$ is the sum of $K$ target echoes.

Then, the definition of $\tau$ is discussed. By assuming that the first element on the right of the equivalent virtual array in the MIMO system is the reference element, denoted by $x_{1}$ and the distance between adjacent elements is $d$, then, the position of the $m$-th elements relative to the reference element is $x_{m}=(m-1) d$, which is shown in Figure 1 . Then, the two-way delay $\tau_{m}$ from the $m$-th element to the target can be expressed as: 


$$
\tau_{m}=\frac{2 R_{k}}{c}+\frac{2 x_{m} \sin \theta_{k}}{c}
$$

where $R_{k}$ is the range from target to reference element and $\theta_{k}$ is the angle of arrival relative to normal.

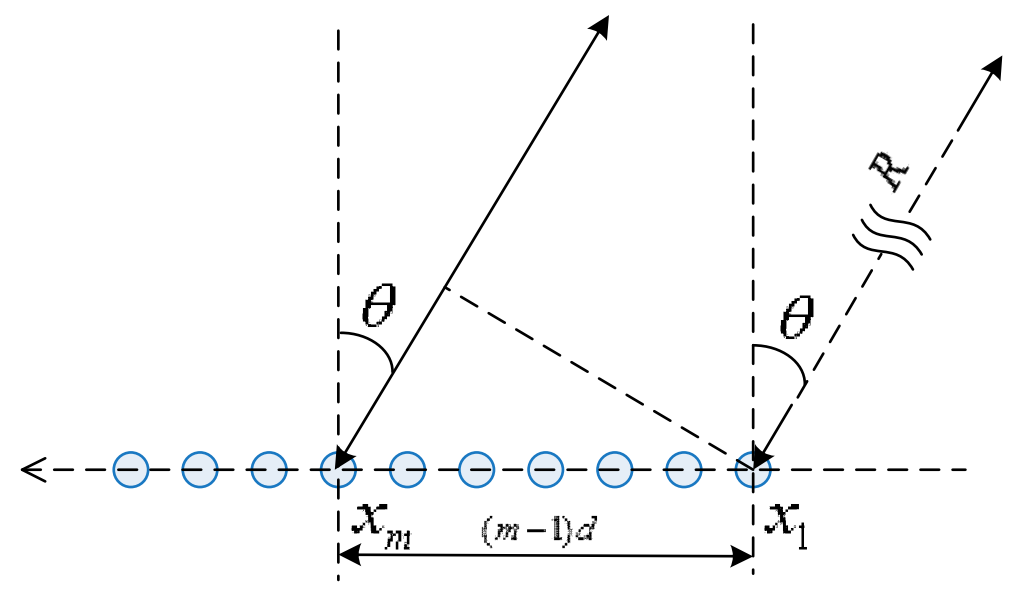

Figure 1. Echo delay diagram.

By combining Equations (7) and (8), the IF signal can be expressed as:

$$
x_{m}(n)=\sum_{k=1}^{K} V_{k} \cos \left(\frac{4 \pi f_{c} x_{m} \sin \theta_{k}}{c}+\frac{4 \pi B T_{s} R_{k} n}{T c}+\frac{4 \pi f_{c} R_{k}}{c}\right)+w_{m}(n), n=0,1,2, \cdots, L-1 .
$$

After performing the signal Hilbert transform on the M IF signal, an analytic signal matrix can be obtained as:

$$
\left[\begin{array}{c}
\mathbf{x}_{1}(n) \\
\mathbf{x}_{2}(n) \\
\vdots \\
\mathbf{x}_{M}(n)
\end{array}\right]=\left[\begin{array}{cccc}
e^{j \frac{4 \pi f_{c} x_{1} \sin \theta_{1}}{c}} & e^{j \frac{4 \pi f_{c} x_{1} \sin \theta_{2}}{c}} & \ldots & e^{j \frac{4 \pi f_{c} x_{1} \sin \theta_{k}}{c}} \\
e^{j \frac{4 \pi f_{c} x_{2} \sin \theta_{1}}{c}} & e^{j \frac{4 \pi f_{c} x_{2} \sin \theta_{2}}{c}} & \ldots & e^{j \frac{4 \pi f_{c} x_{2} \sin \theta_{k}}{c}} \\
\vdots & \vdots & \vdots & \vdots \\
e^{j \frac{4 \pi f_{c} x_{M} \sin \theta_{1}}{c}} & e^{j \frac{4 \pi f_{c} x_{M} \sin \theta_{2}}{c}} & \ldots & e^{j \frac{4 \pi f_{c} x_{M} \sin \theta_{k}}{c}}
\end{array}\right]\left[\begin{array}{c}
V_{1} e^{j\left(\frac{4 \pi B T_{s} R_{1} n}{T c}+\frac{4 \pi f_{c} R_{1}}{c}\right)} \\
V_{2} e^{j\left(\frac{4 \pi B T_{s} R_{2} n}{T c}+\frac{4 \pi f_{c} R_{2}}{c}\right)} \\
\vdots \\
V_{k} e^{j\left(\frac{4 \pi B T_{s} R_{k} n}{T c}+\frac{4 \pi f_{c} R_{k}}{c}\right)}
\end{array}\right]+\left[\begin{array}{c}
w_{1}(n) \\
w_{2}(n) \\
\vdots \\
w_{M}(n)
\end{array}\right] .
$$

It can be rewritten as:

$$
\mathbf{X}=\mathbf{A S}+\mathbf{N}
$$

where $\mathbf{X}$ is the received signal matrix, $\mathbf{N}$ is the noise matrix, $\mathbf{A S}$ is the signal matrix containing the position information of targets, matrix $\mathbf{A}$ contains the angle information of the target, and matrix $\mathbf{S}$ contains the distance information.

\section{MIMO Radar System Implementation}

For this paper, a small FMCW MIMO radar system working in the S-band was implemented. The block diagram is shown in Figure 2. Table 1 shows the main parameters of the proposed MIMO radar system. This radar system can be divided into four parts: the signal generator, the RF front-end, the antenna array, and the IF signal preprocessing circuit. A detailed description of the system block diagram follows. 


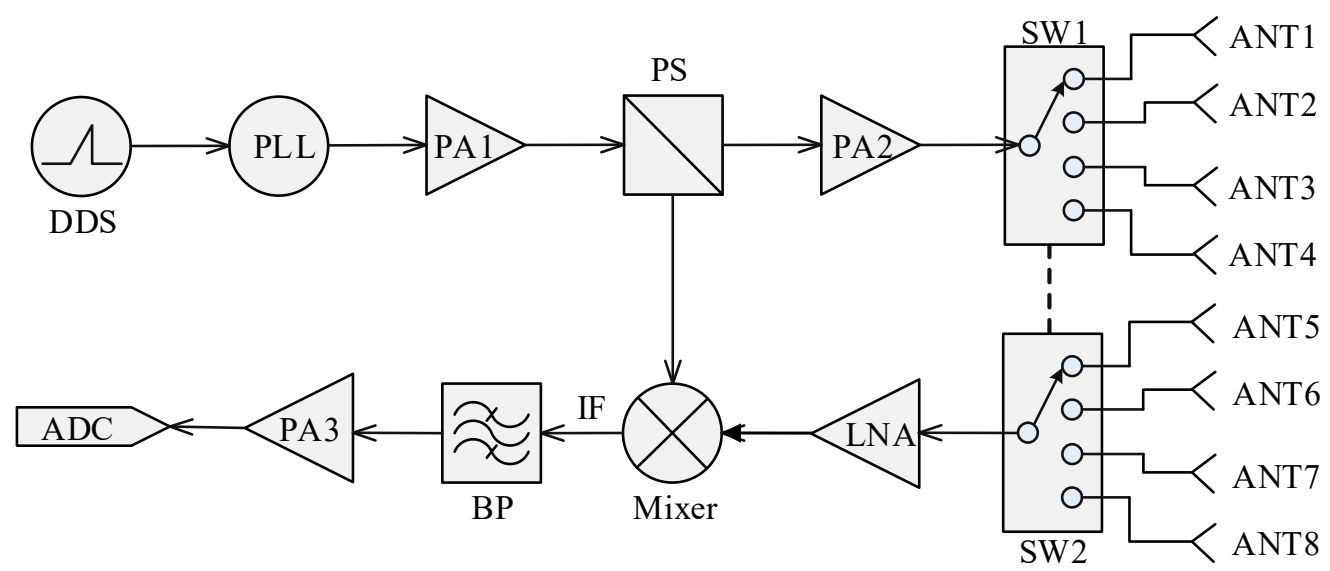

Figure 2. Diagram of the radar system.

Table 1. Parameter of the multiple-input multiple-output (MIMO) radar.

\begin{tabular}{cccc}
\hline Waveform & FMCW & Working mode & TDM \\
\hline Real antenna & $4+4$ & Virtual antenna & 16 \\
\hline Working frequency & $2.46 \mathrm{GHz} 2.54 \mathrm{GHz}$ & Virtual antenna space & $0.08 \mathrm{~m}$ \\
\hline Bandwidth & $80 \mathrm{MHz}$ & Sweep time & $20.6 \mathrm{~ms}$ \\
\hline Transmission power & $18 \mathrm{dBm}$ & ADC sampling rate & $40 \mathrm{kHz}$ \\
\hline
\end{tabular}

\subsection{FMCW Signal Generator}

As the core of this system, a hybrid direct digital synthesizer (DDS) and phase locked loop (PLL) structure was applied to generate the required FMCW signal $[25,26]$. Compared with the traditional mixing method, this method does not produce the image frequencies, so it can generate high quality FMCW signals. Accordingly, no additional filter was required at the signal generator. This specific method uses a DDS with digital-to-analog converter (DAC) function to generate an analog signal whose frequency changes linearly with time, whose phase is controllable, and whose amplitude is adjustable. The start frequency of the chirp signal is $49.2 \mathrm{MHz}$. The bandwidth is $1.6 \mathrm{MHz}$, and the sweep time of one chirp is $20.6 \mathrm{~ms}$. Then, the chirp signal that is generated by the DDS is sent to the PLL. The PLL chip that was used in this system has excellent performance characteristics, such as a high phase detection frequency, short frequency lock time, and extremely low phase noise. Thus, the frequency of the DDS output could be multiplied by 50 times to $2.46-2.54 \mathrm{GHz}$, and the bandwidth becomes $80 \mathrm{MHz}$, which is also the frequency and bandwidth of the transmitted signal.

\subsection{The RF Front End}

The signal from the PLL passes through a power amplifier (PA1) to increase the gain from the original -4 to $12 \mathrm{dBm}$. Thereafter, the signal is passed through a power splitter (PS), where it can be split into two paths: One is amplified and sent to the transmitter, and the other is entered into the mixer as a local oscillator signal. The transmitting signal passes through an adjustable power amplifier (PA2), and the gain parameter is set according to the transmission power requirement. On the other side, the reflected signal from the receiving antenna is mixed with the local oscillator signal after passing through a low noise amplifier (LNA) by the mixer (Mixer). The noise figure of PA1 and LNA is $0.8 \mathrm{~dB}$. In order to reduce the instability of the system, some RF front end components (PA1, PS, LNA and Mixer) are integrated modules that are produced by Mini-Circuits.

The orthogonal transmission waveform of the MIMO radar is achieved by a RF coaxial switch (SW1). This switch turns on the transmitting signal to the four transmitting antennas at different times, and the receiving antennas achieve the echo signals in this way by another RF coaxial switch (SW2). 
In each time period, only one transmitting antenna and one receiving antenna can be connected, so that 16 transmit-receive channels can be formed in one complete cycle. This TDM technique is a relatively simple and general method for implementing an MIMO radar that can greatly reduce the complexity of system design. Figure 3 shows the RF front end.

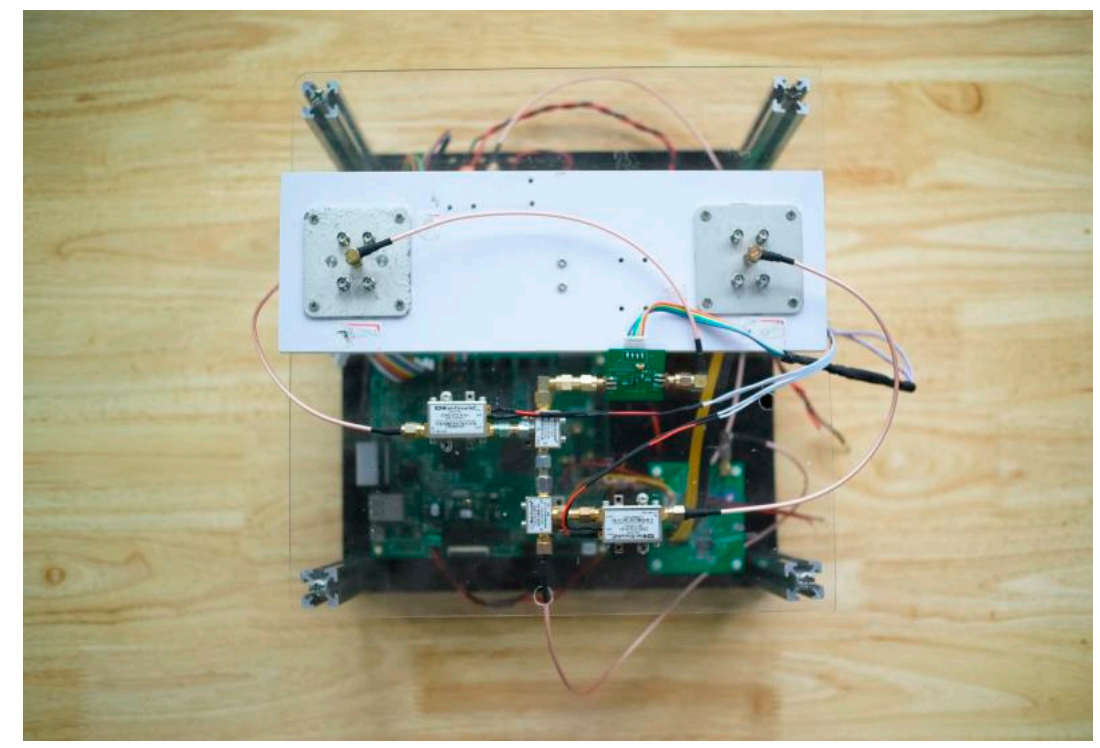

Figure 3. MIMO radar RF front end.

\subsection{The MIMO Antenna Array}

The independent transmitting and receiving antenna elements are used to minimize transmit-to-receive coupling in the MIMO radar system, the schematic of which is shown in Figure 4. The transmitting antenna array on the top row of the diagram is composed of four antennas with unequal spacing (ANT1-4). The receiving antennas are on the bottom row and are composed of four elements with equal spacing (ANT5-8). At any moment, the transmitter and receiver are routed to only one antenna pair. Each pair of transmitting and receiving elements can be independently equivalent to a monostatic element. This equivalent element is marked as the midpoint of the transmit-receive element connection. In this way, 16 effective virtual elements can be obtained and shown in the middle row. The antenna that was used in the system is a flat directional antenna with 4 dipoles that can provide a high RF gain, shown in Figure 5.

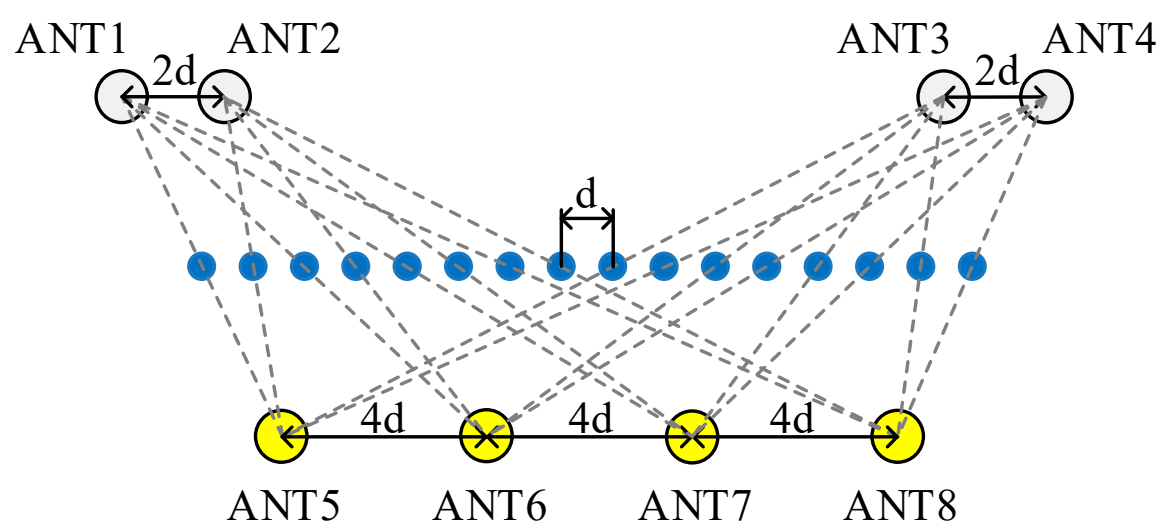

Figure 4. Synthetization of the virtual array. 


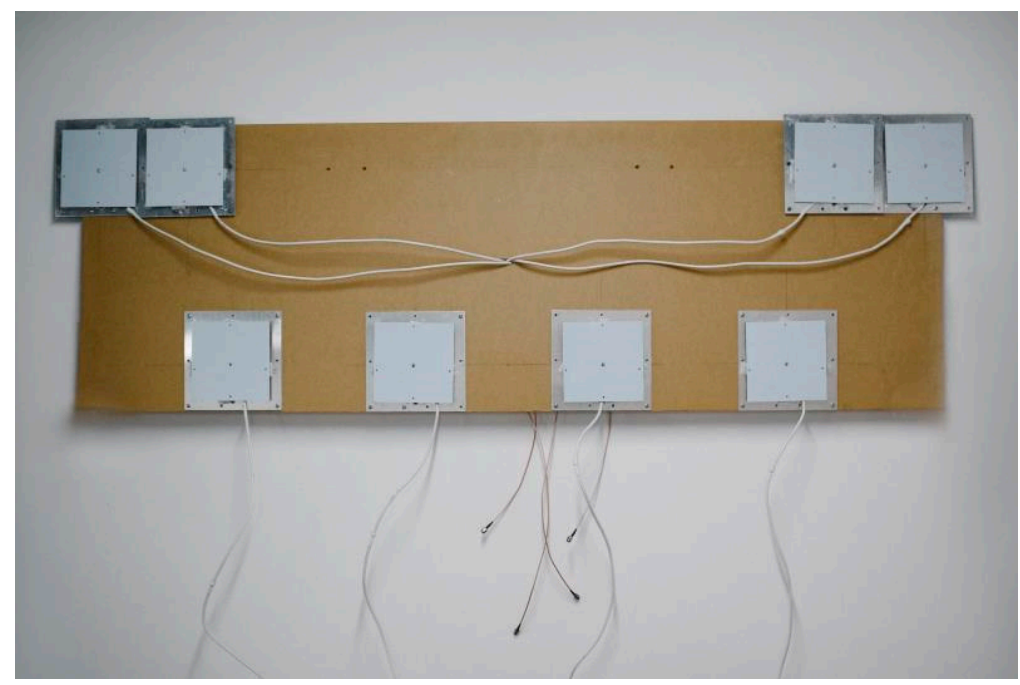

Figure 5. MIMO radar antennas.

\subsection{IF Signal Preprocessing Circuit}

After using the RF switch (SW2) and LNA, the echo signal is mixed with the local oscillator signal to obtain the IF signal. The IF signal needs to be filtered and amplified before discrete sampling. This specific operation was used to design a band-pass filter with an adjustable cut-off frequency. The cut-off frequency of the band-pass filter chip can be changed in order to adjust the input drive frequency so that only the target echo within the desired observation range is passed. The drive frequency of the adjustable filter is provided by an FPGA control board, shown in Figure 6. After the filter, an adjustable gain amplifier (PA3) is used to accommodate the high dynamic range of the radar cross section (RCS). The desired gain amplification can be achieved by adjusting a resistance on the circuit board [27].

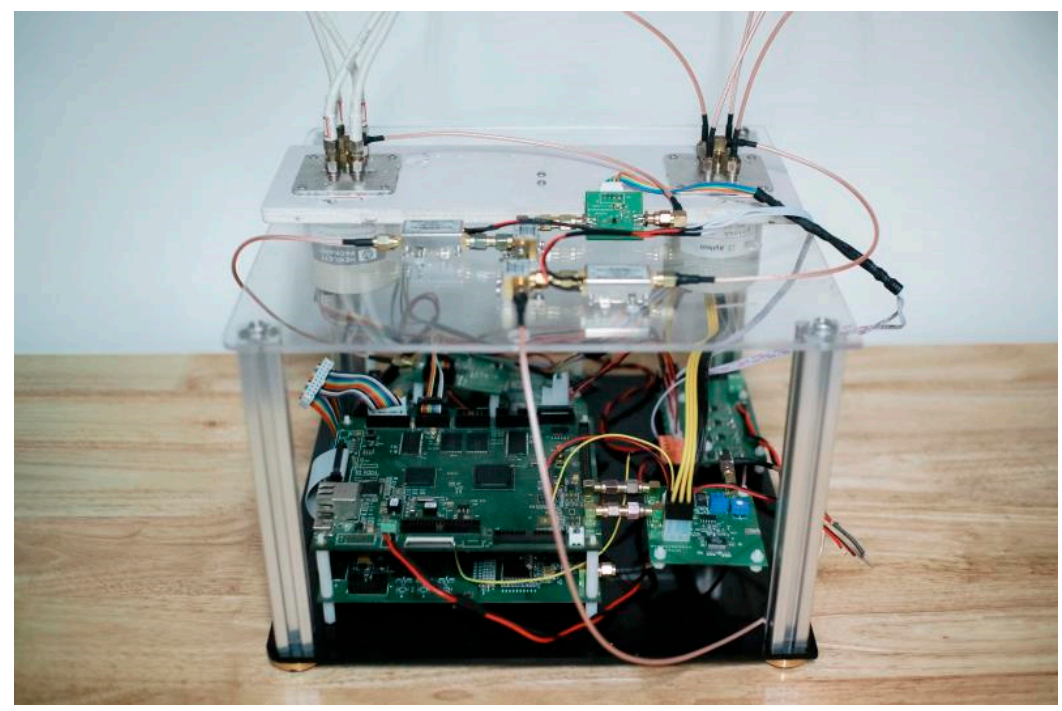

Figure 6. MIMO radar board.

Due to the small sweep slope of the transmitted signal, an analog-to-digital converter (ADC) chip with a low sampling rate was selected to sample the IF signal. For example, if the detected maximum target distance is $500 \mathrm{~m}$, the IF signal frequency that is obtained after mixing is $13000 \mathrm{~Hz}$. Considering the maximum detection range, the actual sampling frequency was set as $40 \mathrm{kHz}$.

The MIMO radar prototype is shown in Figure 7. A complete scan cycle of a radar contains 16 chirps. The time of each chirp is $20.6 \mathrm{~ms}$. During this time, only one set of transmitter-receiving 
antennas is locked by the switch. When a chirp is over, the switch immediately switches to the next transmit-receive channel. When counting the switching time, a complete scan cycle is about $0.6 \mathrm{~s}$.

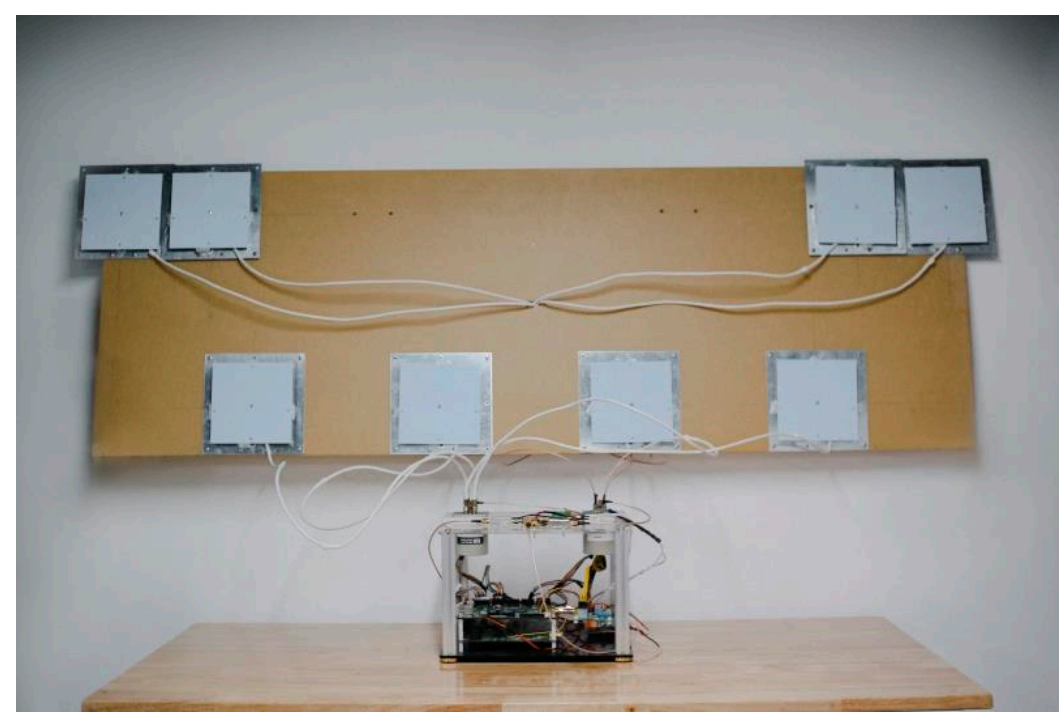

Figure 7. MIMO radar system.

\section{Sparse spectrum fitting Description}

The basic principle of sparse recovery for angle estimation is that the angles of the incident signals have sparsity in the whole space domain. For a ULA, the space domain for angle estimation is [-90 degrees, 90 degrees]. If the appropriate step size is set and the mesh is divided, an over-complete set of angles will be obtained. In this way, the angle estimation problem of the incident signal is transformed into a problem of selecting $\mathrm{K}$ atoms from the angle set and optimizing the corresponding signal amplitude. Similarly, this method also suitable for ranging.

\subsection{Sparse Spectrum Fitting for Angle Estimation}

The covariance of the received data is defined as:

$$
\mathbf{R}_{\mathbf{X}}=E\left(\mathbf{X} \mathbf{X}^{H}\right)=\mathbf{A R}_{\mathbf{S}} \mathbf{A}^{H}+\sigma^{2} \mathbf{I},
$$

where $\mathbf{R}_{\mathbf{S}}$ is the covariance matrix of the signal, $\sigma^{2}$ is the variance of the noise, and $\mathbf{I}$ is the identity matrix. Considering the actual received data matrix with finite snapshots, the maximum likelihood estimation of the covariance matrix is:

$$
\hat{\mathbf{R}_{\mathbf{X}}}=\frac{1}{L} \mathbf{X} \mathbf{X}^{H} .
$$

Assuming the targets are uncorrelated, there is a vectorized covariance matrix:

$$
\mathbf{R}_{v}=\mathbf{A}_{J} p^{\circ}+\mathbf{E}_{v}
$$

where $J\left(>>K\right.$ and $\left.>\mathrm{M}^{2}\right)$ is the number of grids divided by the angle of the expected estimate, and:

$$
\begin{gathered}
\mathbf{E}_{v}=\operatorname{vec}\left(\sigma^{2} \mathbf{I}\right), \\
\mathbf{A}_{J}=\left[a_{v}\left(\theta_{1}\right), a_{v}\left(\theta_{2}\right), \cdots, a_{v}\left(\theta_{J}\right)\right] \in C^{M^{2} \times J}, \\
a_{v}(\theta)=\operatorname{vec}\left(a(\theta) a^{H}(\theta)\right) \in C^{M^{2} \times 1}, \\
p^{\circ}=\left[p_{1}^{\circ}, p_{2}^{\circ}, \cdots, p_{J}^{\circ}\right]^{T}=\operatorname{diag}\left(R_{S}\right) \in R_{+}^{J \times 1},
\end{gathered}
$$


When the number of snapshots is large enough, there is a convex optimization, which has the following form:

$$
\begin{array}{r}
p *=\underset{p}{\operatorname{argmin}}\left\|\mathbf{R}_{v}-\mathbf{A}_{J} p\right\|_{2}^{2}+\alpha\|p\|_{1} \\
\text { s.t. } p_{i} \geq 0, \quad i=1,2, \cdots, J
\end{array}
$$

where $p^{*}$ is the optimal vector and $\alpha$ is the regularization parameter, which can be determined by the chi-square distribution and noise power. When the number of snapshots tends to infinity, $\alpha$ tends to $\sigma^{2} \mathbf{I}$.

\subsection{Sparse Spectrum Fitting for Range Estimation}

It can be seen from Equation (10) that vector $\mathbf{S}$ contains the distance information of the target, so one must use the SpSF method to estimate the distance required for the space sequence matrix as the body of the signal.

In Equation (11), the transpose of the received signal matrix is shown as:

$$
\mathbf{X}^{T}=\mathbf{S}^{T} \mathbf{A}^{T}+\mathbf{N}^{T} .
$$

Its covariance is:

$$
\mathbf{R}_{T \mathbf{X}}=E\left(\mathbf{X}^{T}\left(\mathbf{X}^{T}\right)^{H}\right)=\mathbf{S}^{T} \mathbf{R}_{T \mathbf{S}}\left(\mathbf{S}^{T}\right)^{H}+\sigma^{2} \mathbf{I} .
$$

The maximum likelihood estimate of the covariance matrix is:

$$
\hat{\mathbf{R}}_{T \mathbf{X}}=\frac{1}{M} \mathbf{X}^{T}\left(\mathbf{X}^{T}\right)^{H} .
$$

Then, the desired range of the estimated distance is set to a suitable step size and meshed, and an ultra-complete range set is obtained. The estimation method is similar to that of angle estimation and is thus not repeated here.

\section{Simulated and Experimental Results}

For this section, the SpSF algorithm was used to test the simulation data and real data achieved by the designed FMCW MIMO radar, and the performance of the algorithm is compared with the MUSIC algorithm and the Capon algorithm.

\subsection{Simulated Results}

In order to more intuitively compare the algorithm performance, the MIMO radar parameters in the simulation were the same as those introduced in Section 3. With a sampling frequency of $40 \mathrm{kHz}$, 824 discrete data could be sampled in a chirp duration. Suppose that are there are three targets in the scenario. The specific parameters are shown in the Table 2 below. Gaussian white noise was added to the simulation data.

Table 2. Simulated target parameters.

\begin{tabular}{ccc}
\hline & Angle (degree) & Range (m) \\
\hline Target1 & -10.1 & 30.1 \\
Target2 & 2.3 & 40.3 \\
Target3 & 5.5 & 50.5 \\
\hline
\end{tabular}

Firstly, the angle of the target was estimated, and the results are shown in Figure 8: Figure 8a,c are the estimated angles of targets when using all the sampling points (824 points) of a chirp cycle, while Figure $8 \mathrm{~b}, \mathrm{~d}$ are estimated angles of the targets when using the first 100 sampling points of a chirp cycle. The SNR of Figure $8 a, b$ is $0 d B$, and that of Figure $8 c, d$ is $-10 d B$. 


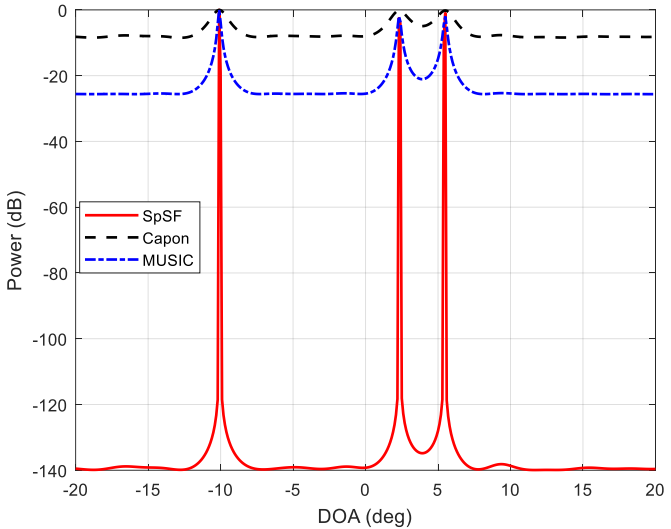

(a) Snapshots $=824, \mathrm{SNR}=0 \mathrm{~dB}$

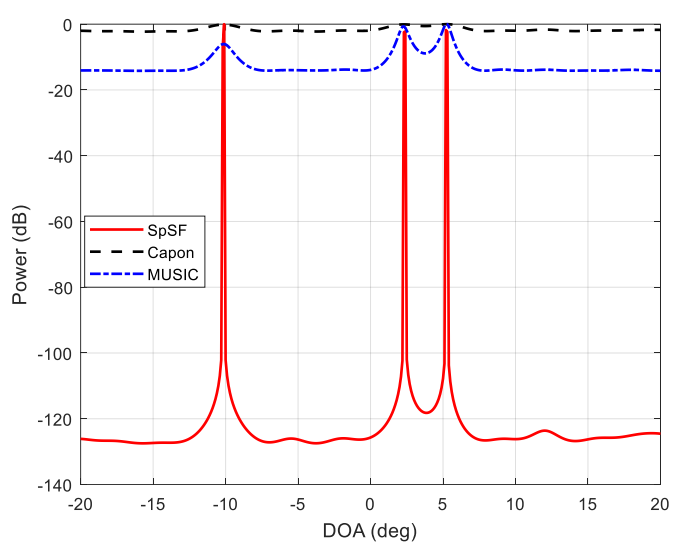

(c) Snapshots $=824, \mathrm{SNR}=-10 \mathrm{~dB}$

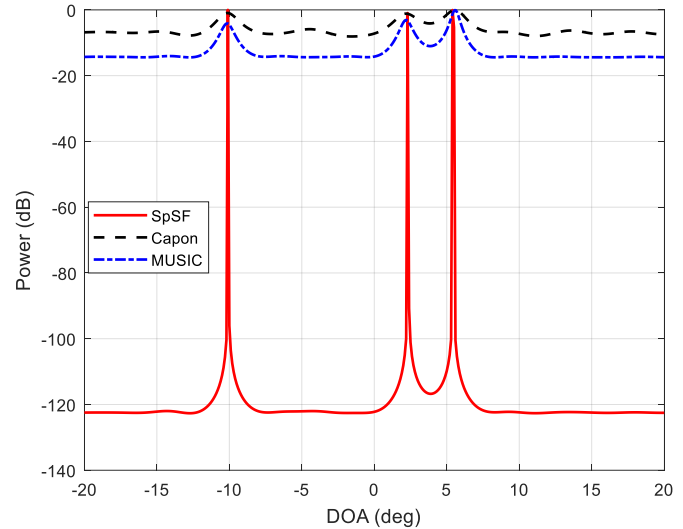

(b) Snapshots $=100, \mathrm{SNR}=0 \mathrm{~dB}$

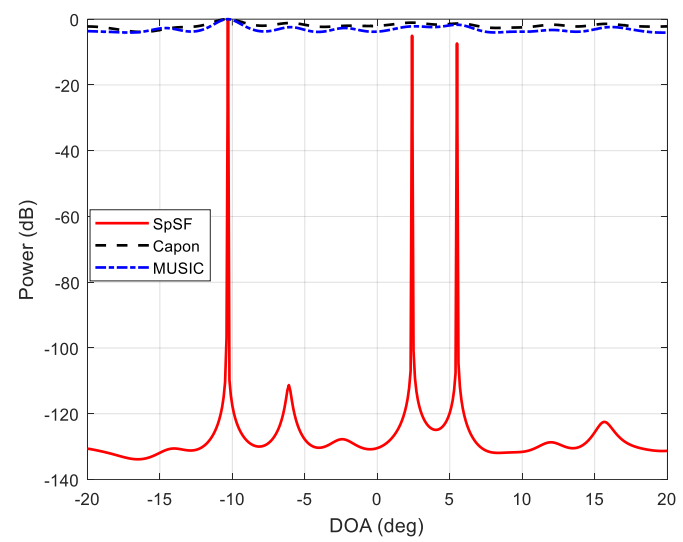

(d) Snapshots $=100, \mathrm{SNR}=-10 \mathrm{~dB}$

Figure 8. Angle estimation of the simulated data.

Compared with the Capon and MUSIC algorithms, it can be seen that in the case of a high SNR and large snapshots, these three algorithms could accurately estimate the angles of the three targets. However, with the decrease of the SNR, the performance of the Capon and MUSIC algorithms declined under different conditions. Especially in the case of a small number of snapshots, the performance significantly deteriorated and the angles of different targets could be hardly distinguished. However, the SpSF algorithm had an excellent estimation performance, even in the case of a low SNR and small snapshots.

Next, the distance of the targets based on simulated data was estimated. Since the estimation of distance is obtained by transposing a received matrix, when the number of snapshots is too large, the calculation amount explodes, which is not desirable to practical application. Thus, simulated data with 100 snapshots were used to estimate the distance. Figure 9 shows the estimated results when the SNR was 20,10 , and $0 \mathrm{~dB}$, respectively.

It can be seen from the simulation results that when the number of snapshots was constant, the estimation performance of the Capon and MUSIC algorithms significantly deteriorated with the decrease of the SNR. When the SNR was $0 \mathrm{~dB}$, the MUSIC and Capon algorithms could no longer distinguish the distance of the targets, while the SpSF algorithm still maintained an excellent estimation performance. 


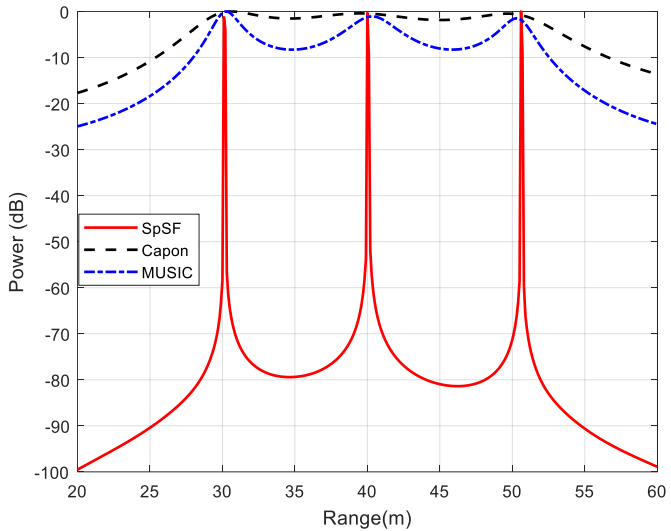

(a) $\mathrm{SNR}=20 \mathrm{~dB}$

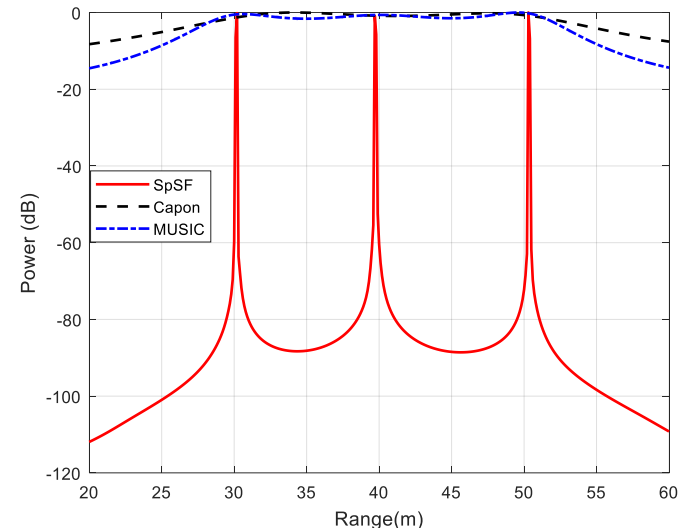

(b) $\mathrm{SNR}=10 \mathrm{~dB}$

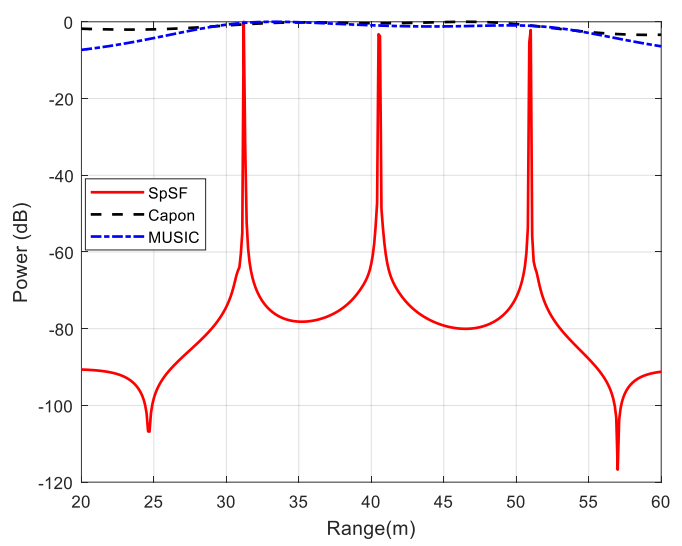

(c) $\mathrm{SNR}=0 \mathrm{~dB}$

Figure 9. Range estimation of the simulated data.

\subsection{Field Experimental Results}

The experimental data were obtained by the FMCW MIMO radar system that was introduced in Section 3. The experimental site was an empty field with three highlight metal reflectors whose location is shown in Figure 10. The distance from the target to the antennas was measured by using a tape, and the angle was calculated by triangle conversion. The specific parameters are shown in Table 3 below.

In the experiment, the maximum angle of the target relative to the center of the antenna array was 12.4 degrees, so the estimated range was selected as [ -20 degrees, 20 degrees], and the step size was set to 0.1 degrees. For the distance, the distance between the target and the antenna should not exceed $50 \mathrm{~m}$. Thus, the estimated range was chosen to be $[20 \mathrm{~m}, 60 \mathrm{~m}]$, and the step size was set to $0.1 \mathrm{~m}$. The echo signals outside the desired detection range were filtered out by the IF preprocessing circuit in the radar system before ADC sampling so that the reflected interference of distant buildings and trees was avoided. Figure 11 shows the angle estimation results of the received data when the number of snapshots was 824 and 100. All three algorithms could estimate the angle of targets well in the case of large snapshots. Through the detailed comparison of the spectral peaks that were estimated by the three algorithms, the error could be seen as being within 0.1 degrees. In the case of the small snapshots, the performance of the three methods decreased to different degrees, among which the comparison method could not normally distinguish the angles of the three targets, while the SpSF algorithm could estimate the angles and have good performance. 


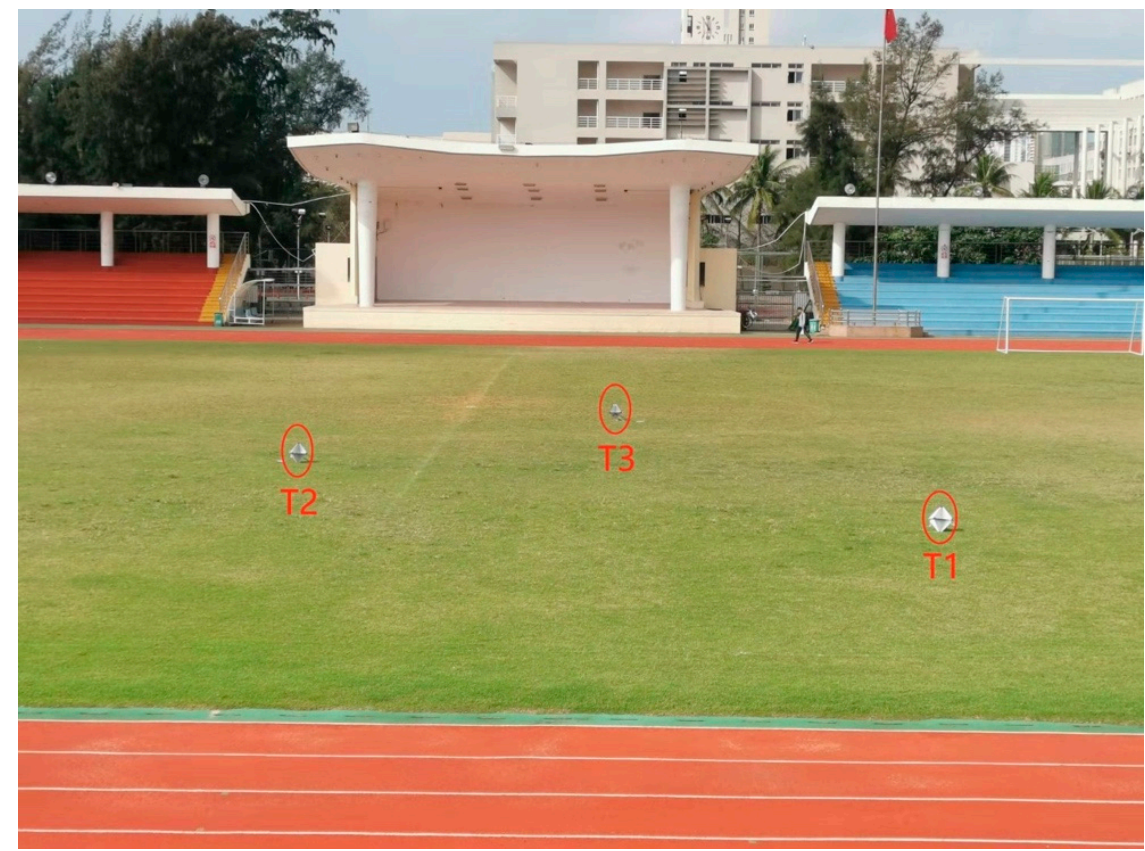

Figure 10. Scenario for the measured data set collection with 3 highlighted targets.

Table 3. Actual target parameters of 3 targets.

\begin{tabular}{ccccc}
\hline & Range_X $(\mathbf{m})$ & Range_Y $\left._{\mathbf{( m}}\right)$ & Range $(\mathbf{m})$ & Angle (degree) \\
\hline Target1(T1) & 6.7 & 30.5 & 31.2 & 12.4 \\
\hline Target2(T2) & -5.8 & 38.6 & 39.1 & -8.5 \\
\hline Target3(T3) & 0.3 & 48.5 & 48.5 & 0.4 \\
\hline
\end{tabular}

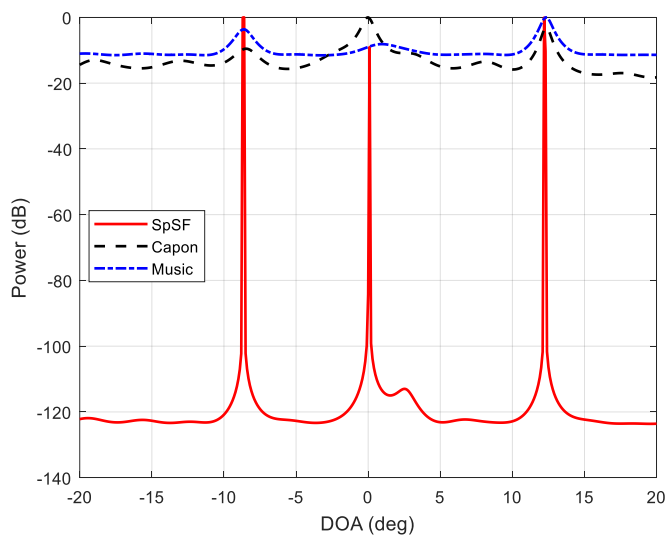

(a) Snapshots $=824$

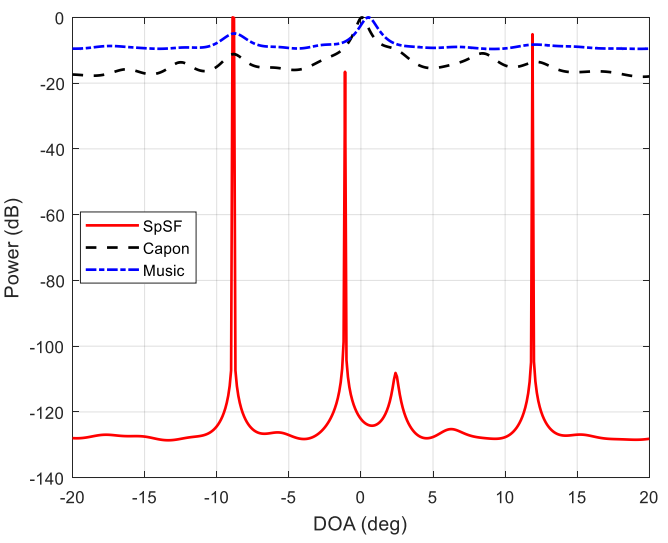

(b) Snapshots $=100$

Figure 11. Angle estimation of the real data.

One hundred snapshots were selected to estimate the distance of the target. As can be seen from the results in Figure 12, in the case of the MUSIC and Capon algorithms, only two slightly larger spectral peaks could be seen, which indicates that they failed to work. However, the SpSF algorithm could accurately estimate the three target distances with an error range of $0.5 \mathrm{~m}$. 


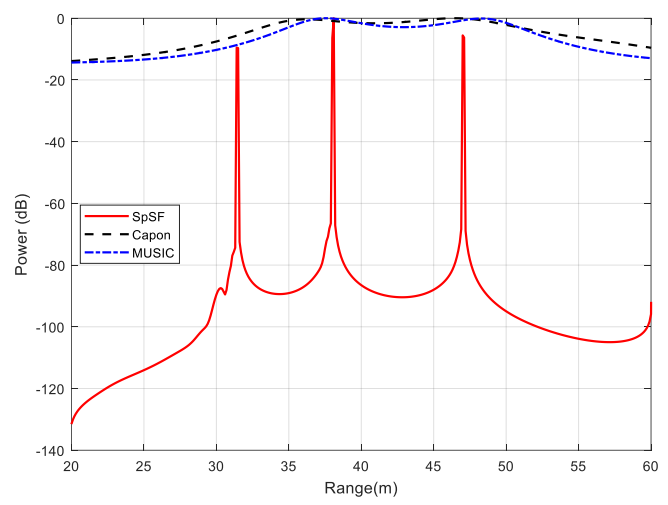

Figure 12. Range estimation of the real data.

The experimental scenario that was used to perform the experimental results of the two targets is shown in Figure 13, and the experimental results are shown in Figure 14.

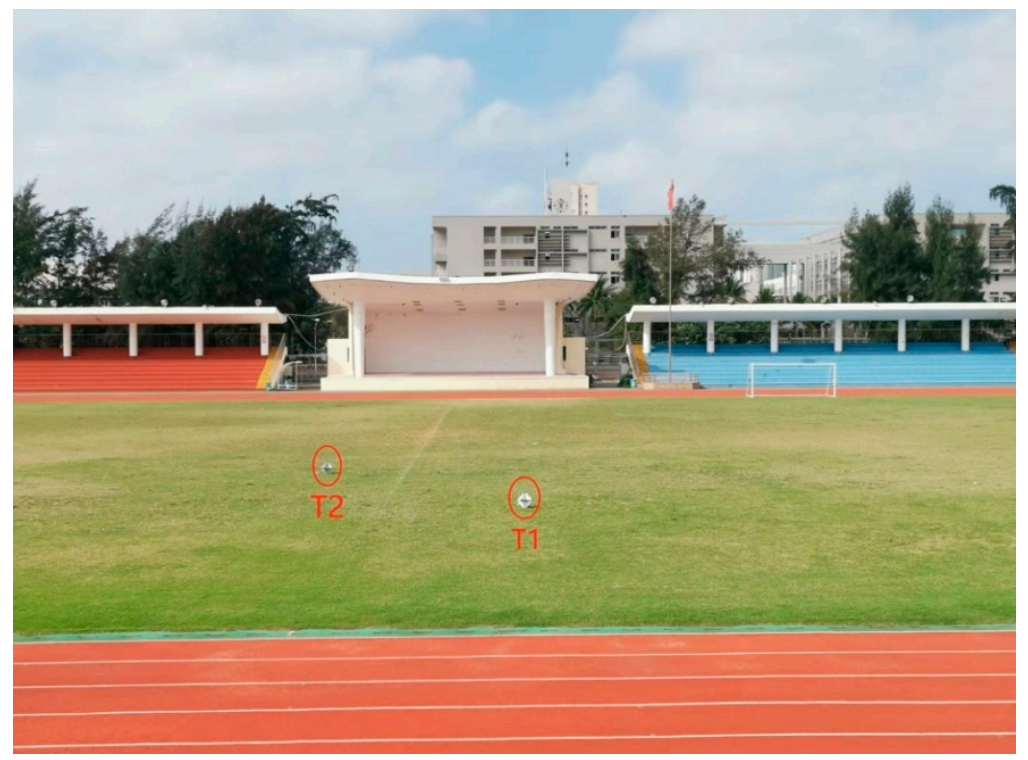

Figure 13. Scenario for the measured data set collection with 2 highlighted targets.

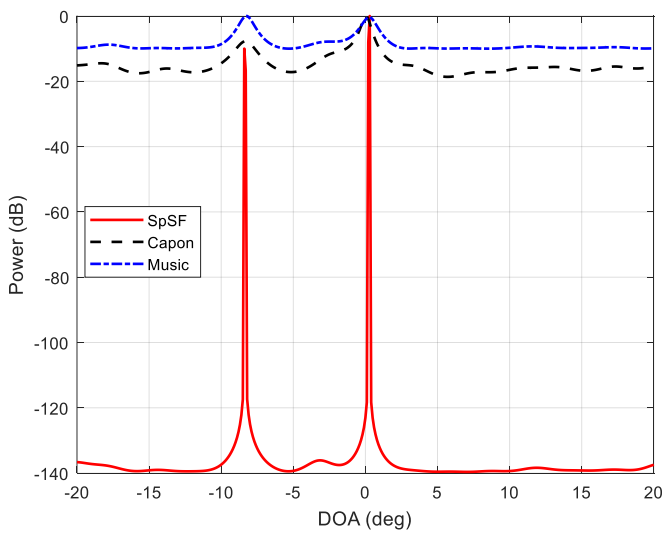

(a) Angle estimation of two targets

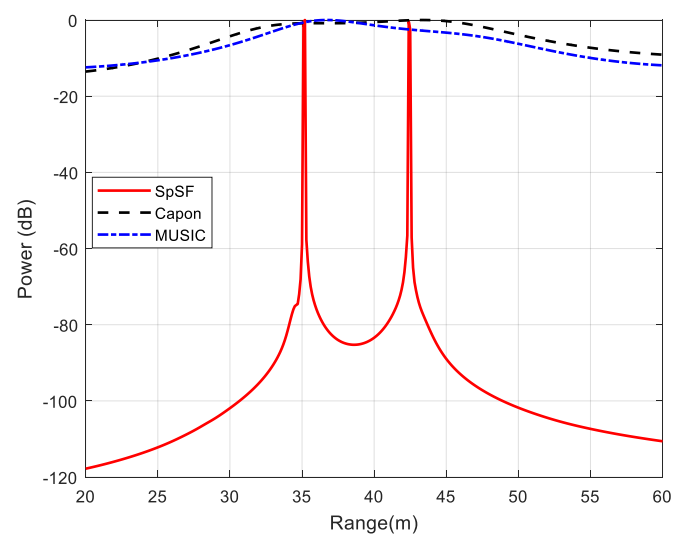

(b) Range estimation of two targets

Figure 14. Estimation of two targets. 
The distance and the angle of target1 (T1) were $34.4 \mathrm{~m}$ and 0.3 degrees, respectively; the distance and the angle of target2 (T2) were $41.8 \mathrm{~m}$ and -7.9 degrees, respectively. It can be seen from the experimental results that the SpSF algorithm had a superior performance, whether for the angle estimation or distance estimation of the targets, as shown in Figure 14.

\section{Conclusions}

In this paper, an FMCW MIMO radar system that used the TDM method was designed, its initial prototype was implemented, and a sparse spectrum fitting (SpSF) algorithm was formulated for estimating the distance and angle. A frequency multiplication structure, which can avoid the appearance of imaging frequency, was designed as the FMCW signal generator in the radar transceiver. The echo signals outside the desired observation range can be filtered by the filter circuit, and the amplitude of different RCSs can be adjusted by the amplifier. The capability and performance of the radar system was verified by formulating the SpSF algorithm via field experiments. The simulated and experimental results proved the effectiveness of the designed MIMO radar and the superior performance of the SpSF algorithm. However, the SpSF algorithm has not been run on a system board. The next step of our work is to run the algorithm on this system board and to optimize the system switching time to achieve the real-time estimation of the target.

Author Contributions: L.H. wrote the paper. X.W. and M.H. proposed the original idea. Z.H. and Y.Y. designed the simulations and analyzed the data, and L.W. checked the paper. All authors have read and agreed to the published version of the manuscript.

Funding: This work was funded by Key Research and Development Program of Hainan Province (No. ZDYF2019011), the National Natural Science Foundation of China (No. 61701144, No. 61961013, No. 61861015), the Program of Hainan Association for Science and Technology Plans to Youth R\&D Innovation (No. QCXM201706), the scientific research projects of University in Hainan Province (No. Hnky2018ZD-4), Young Elite Scientists Sponsorship Program by CAST (No. 2018QNRC001), Collaborative Innovation Fund of Tianjin University and Hainan University (No. HDTDU201906), and the Scientific Research Setup Fund of Hainan University (No. KYQD (ZR) 1731).

Conflicts of Interest: The authors declare no conflict of interest.

\section{References}

1. Fishler, E.; Haimovich, A.; Blum, R.; Chizhik, D.; Cimini, L.; Valenzuela, R. MIMO radar: An idea whose time has come. In Proceedings of the 2004 IEEE Radar Conference, Philadelphia, PA, USA, 29 April 2004.

2. Wang, H.; Wan, L.; Dong, M.; Ota, K.; Wang, X. Assistant vehicle localization based on three collaborative base stations via SBL-based robust DOA estimation. IEEE Internet Things J. 2019, 6, 5766-5777. [CrossRef]

3. Wang, X.; Wan, L.; Huang, M.; Shen, C.; Zhang, K. Polarization Channel Estimation for Circular and Non-Circular Signals in Massive MIMO Systems. IEEE J. Sel. Top. Signal Process. 2019, 13, 1001-1016. [CrossRef]

4. Wang, X.; Wan, L.; Huang, M.; Shen, C.; Han, Z.; Zhu, T. Low-Complexity Channel Estimation for Circular and Noncircular Signals in Virtual MIMO Vehicle Communication Systems. IEEE Trans. Veh. Technol. 2020, 1. [CrossRef]

5. Liu, Y.; Deng, Y.K.; Wang, R.; Loffeld, O. Bistatic FMCW SAR signal model and imaging approach. IEEE Trans Aerosp. Electron. Syst. 2013, 49, 2017-2028. [CrossRef]

6. Pfeffer, C.; Feger, R.; Wagner, C.; Stelzer, A. FMCW MIMO Radar System for Frequency-Division Multiple TX-Beamforming. IEEE Trans. Microw. Theory Tech. 2013, 61, 4262-4274. [CrossRef]

7. Belfiori, F.; Mass, N.; Hoogeboom, P.; van Rossum, W. TDMA X-band FMCW MIMO radar for short range surveillance applications. In Proceedings of the 5th European Conference on Antennas and Propagation (EUCAP), Rome, Italy, 11-15 April 2011.

8. Chen, H.; Li, X.; Jing, W.; Zhuang, Z. MIMO radar sensitivity analysis of antenna position for direction finding. IEEE Trans. Signal Process. 2012, 60, 5201-5216. [CrossRef]

9. Wang, J.; Jiang, S.; He, J.; Liu, Z.; Baker, C.J. Adaptive subspace detector for multi-input multi-output radar in the presence of steering vector mismatch. IET Radar Sonar Navig. 2009, 5, 23-31. [CrossRef] 
10. Li, H.; Himed, B. Transmit subaperturing for MIMO radars with co-located antennas. IEEE J. Sel. Topics Signal Process. 2010, 4, 55-65. [CrossRef]

11. Charvat, G.L.; Kempel, L.C.; Rothwell, E.J.; Coleman, C.M.; Mokole, E.L. A through-dielectric ultrawideband (UWB) switched-antenna-array radar imaging system. IEEE Trans Antennas Propag. 2010, 60, 5195-5500. [CrossRef]

12. Frazer, G.J.; Abramovich, Y.I.; Johnson, B.A.; Robey, F.C. Recent results in MIMO over-the-horizon radar. In Proceedings of the 2008 IEEE Radar Conference, Rome, Italy, 26-30 May 2008.

13. Feger, R.; Wagner, C.; Schuster, S.; Scheiblhofer, S.; Jager, H.; Stelzer, A. A 77-GHz FMCW MIMO radar based on an SiGe single-chip transceiver. IEEE Trans. Microwave Theory Tech. 2009, 57, 1020-1035. [CrossRef]

14. Ng, H.J.; Kissinger, D. Highly Miniaturized 120-GHz SIMO and MIMO Radar Sensor with On-Chip Folded Dipole Antennas for Range and Angular Measurements. IEEE Trans. Microwave Theory Tech. 2018, 66, 2592-2603. [CrossRef]

15. Richards, M.A. Iterative Noncoherent Angular Superresolution. In Proceedings of the 1988 IEEE National Radar Conference, Ann Arbor, MI, USA, 20-21 April 1988.

16. Levanon, N.; Mozeson, E. Radar Signals, 3rd ed.; John Wiley \& Sons Inc: Hoboken, NJ, USA, 2004.

17. Belfiori, F.; van Rossum, W.; Hoogeboom, P. 2D-MUSIC technique applied to a coherent FMCW MIMO radar. In Proceedings of the IET International Conference on Radar Systems (Radar 2012), Glasgow, UK, 22-25 October 2012.

18. Xie, N.; Guo, W.B.; Zhang, L. High Resolution DOA Estimation Method in MIMO Radar. Wirel. Pers. Commun. 2016, 91, 219-236. [CrossRef]

19. Kim, S.; Yu, J.; Jeon, S. Signal Processing for a Multiple-Input Multiple-Output (MIMO) Video Synthetic Aperture Radar (SAR) with Beat Frequency Division Frequency-Modulated Continuous Wave (FMCW). Remote Sens. 2017, 9, 491. [CrossRef]

20. Li, J.; Jin, M.; Zhang, Y.; Liao, G.; Li, L. Transmit and receive array gain-phase error Estimation in bistatic MIMO radar. IEEE Antennas Wirel. Propag. Lett. 2015, 14, 32-35. [CrossRef]

21. Pan, M.; Chen, B. MIMO High Frequency Surface Wave Radar Using Sparse Frequency FMCW Signals. Int. J. Antennas Propag. 2017, 2017, 1-16. [CrossRef]

22. Wang, X.; Wang, W.; Li, X.; Liu, J. Real-valued covariance vector sparsity-inducing DOA estimation for monostatic MIMO radar. Sensors 2015, 15, 28271-28286. [CrossRef] [PubMed]

23. Zheng, J.; Kaveh, M. Sparse spatial spectral estimation: A covariance fitting algorithm, performance and regularization. IEEE Trans. Signal Process. 2013, 61, 2767-2777. [CrossRef]

24. Zheng, J.; Kaveh, M. Sparse spectral fitting for Direction of Arrival and power estimation. IEEE Signal Process. Lett. 2015, 22, 435-439.

25. Zhao, Z.Y.; Li, X.Y.; Chang, W.G. LFM-CW signal generatorbased on hybrid DDS-PLL structure. Electron. Lett. 2013, 49, 391-393. [CrossRef]

26. Bonfanti, A.; Amorosa, F.; Somori, C.; Lacaita, A.L. A DDS-based PLL for 2.4-GHz frequency synthesis. IEEE Trans. Circuits Syst. II Analog Digital Signal 2003, 50, 1007-1010. [CrossRef]

27. Grillo, G.J.; Perez, M.A.; Florencias, A.E. Synchronic filter based on switched capacitor filters for high stability phase-fetectors systems. In Proceedings of the 2006 IEEE Instrumentation and Measurement Technology Conference, Sorrento, Italy, 24-27 April 2006.

(C) 2020 by the authors. Licensee MDPI, Basel, Switzerland. This article is an open access article distributed under the terms and conditions of the Creative Commons Attribution (CC BY) license (http://creativecommons.org/licenses/by/4.0/). 